
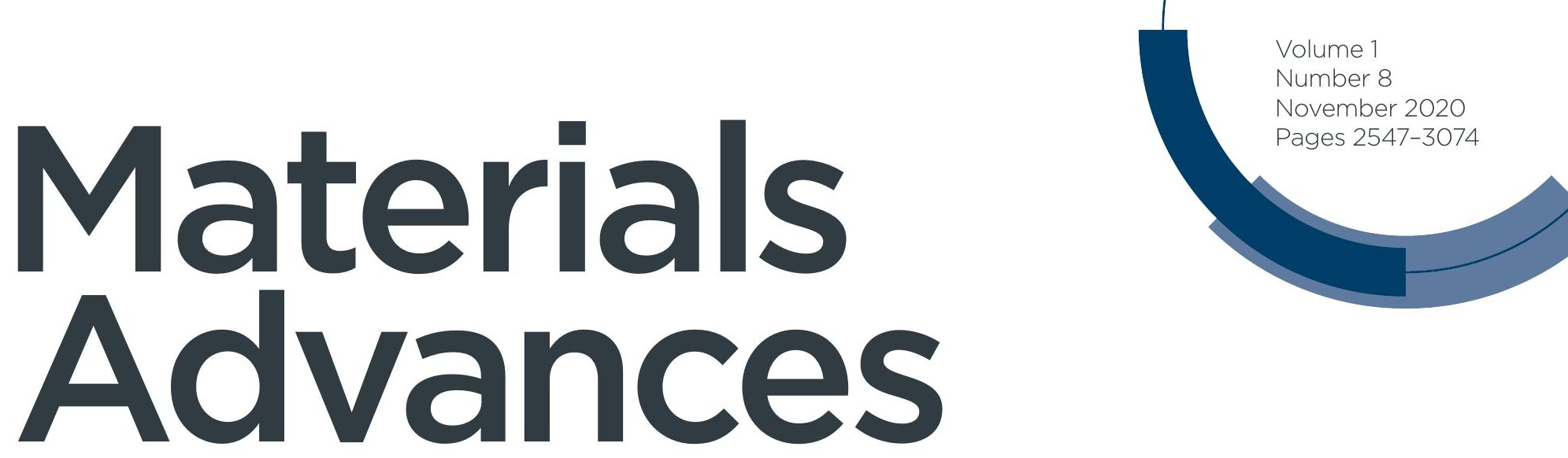

rsc.li/materials-advances

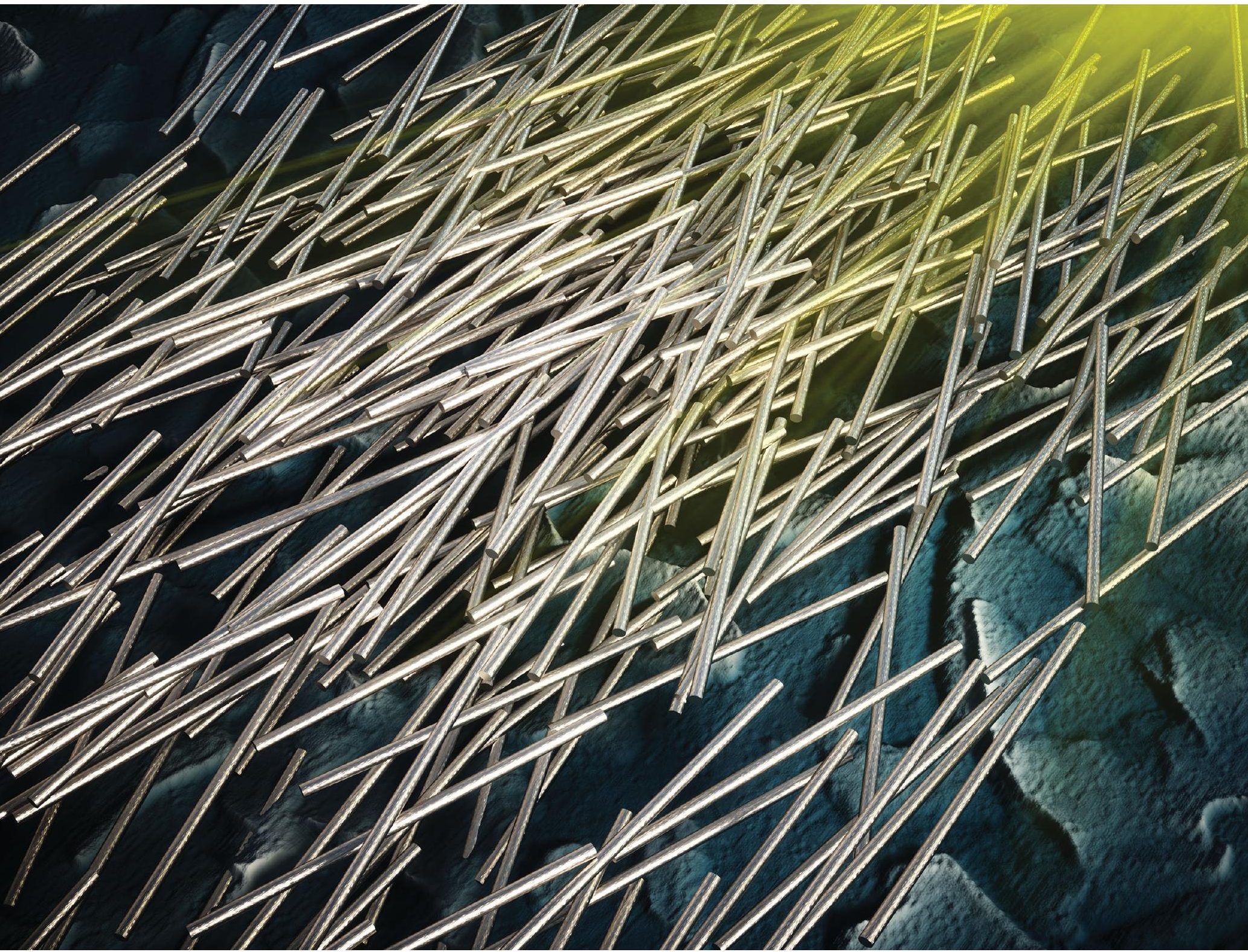

ISSN 2633-5409

ROYAL SOCIETY

$\longrightarrow$ OF CHEMISTRY

\section{PAPER}

Randy J. Ellingson et al.

Aspect ratio controlled synthesis of tellurium nanowires for

photovoltaic applications 
Check for updates

Cite this: Mater. Adv., 2020 1,2721

Received 9th June 2020

Accepted 10th August 2020

DOI: 10.1039/d0ma00394h

rsc.li/materials-advances

\title{
Aspect ratio controlled synthesis of tellurium nanowires for photovoltaic applications
}

\author{
Dipendra Pokhrel, (D) Ebin Bastola, (D) Adam B. Phillips, (D) Michael J. Heben (D) and \\ Randy J. Ellingson (D) *
}

\begin{abstract}
Here, we report an aspect ratio-controlled synthesis of tellurium (Te) nanowires (NWs) utilizing a hotinjection colloidal method. The synthetic method uses low cost materials - specifically, tellurium oxide $\left(\mathrm{TeO}_{2}\right)$ as the tellurium source, environmentally benign ethylene glycol as a solvent, and ascorbic acid as a reducing agent. Analysis of scanning electron micrographs confirms that the NWs show narrow distributions for length, diameter, and therefore also aspect ratio. For example, we have prepared Te NWs exhibiting aspect ratios of 28.0 and 55.4. X-ray diffraction studies confirm a hexagonal crystal structure for the synthesized Te NWs. Based on Raman spectroscopy, Stokes lines were observed at 117.0 and $136.1 \mathrm{~cm}^{-1}$ for $A_{1}$ and $E_{2}$ modes of vibration. Fabrication of Te NW thin films demonstrated their suitability as a hole transport layer at the back contact of cadmium sulfide/cadmium telluride (CdS/CdTe) solar cells, yielding enhanced open-circuit voltage $\left(V_{\text {oc }}=0.846 \mathrm{~V}\right)$ and fill factor (FF $=77.8 \%$ ), and an efficiency of $14.6 \%$; the control device has an efficiency of $13.1 \%\left(V_{\text {oc }}=0.825 \mathrm{mV}, \mathrm{FF} 74.0 \%\right)$
\end{abstract}

\section{Introduction}

Nanomaterials offer significant value for opto-electronic applications because of their unique optical and electronic properties compared to their bulk counterparts. ${ }^{1-3}$ Properly prepared onedimensional semiconductors, such as nanowires (NWs), possess superior axial transport properties (thermal and electrical conductivity) which make them applicable in opto-electronics including nano photonics and energy related applications. ${ }^{4,5}$ Tellurium (Te) is a rare earth element with narrow band gap energy $(\sim 0.35 \mathrm{eV})$ and p-type conductivity. ${ }^{6}$ Te NWs often exhibit low defect density, enhanced thermoelectric performance, and photoconductivity. ${ }^{7-10}$ Here, we report on success in preparing controlled aspect ratio Te NWs, and demonstrate their use as a buffer layer for the back contact of cadmium telluride (CdTe) photovoltaic (PV) devices.

Although Te nanostructures such as nanowires, nanorods and nanobelts can be prepared using solution-based and vacuum based approaches, these methods typically yield product with a wide size polydispersity, particularly in length. Solutionbased techniques include solvothermal and hydrothermal approaches ${ }^{11-17}$ whereas for vacuum based technology include vapor deposition of Te such as thermal evaporation. ${ }^{18,19}$ Mohanty et al. have used thermal evaporation to produce Te NWs of several micron length with diameters of $100-300 \mathrm{~nm} \cdot{ }^{19}$ The solution-

Wright Center for Photovoltaics Innovation and Commercialization, Department of Physics and Astronomy, The University of Toledo, Toledo, OH, 43606, USA.

E-mail: Randy.Ellingson@utoledo.edu based approaches include the use of hydrazine as a reducing agent ${ }^{14,15,20}$ which is extremely hazardous and highly flammable. Thus, less hazardous and safer chemicals are desired for the synthesis of Te NWs. The solution-based synthesis gives a freedom for the solution-processing of the material for the fabrication of devices. Silva et al. yield the Te NWs with diameter less than $30 \mathrm{~nm}$ and lengths varying from $600 \mathrm{~nm}$ to $5 \mu \mathrm{m}$ using hypophosphorous acid. ${ }^{21}$ Liu et al. have reported the rapid microwave assisted method to produce Te NWs with $20 \mathrm{~nm}$ diameter and length of tens of microns. ${ }^{8}$ In the synthesis of nanorods and nanowires, ascorbic acid is used as a reducing agent which is relatively less hazardous. ${ }^{11,22-24}$ Yang et al. reported the synthesis of Te NWs using ascorbic acid as a reducing agent and ethylene glycol as the solvent with average diameter of $9.7 \pm 2.7 \mathrm{~nm}^{24}$ Based on the literature, all these Te NWs have relatively uniform diameters but wide distribution of lengths. Uniform and controlled length of these Te NWs would provide an opportunity to improve the transport properties along the length. Thus, our novel strategy focuses on the synthesis of Te NWs with controlled aspect ratio, for application at the back-contact in CdTe solar cells.

Cadmium telluride is one of the leading cost-effective PV technologies for thin film solar cells with direct band gap energy of $1.5 \mathrm{eV}$ and absorption coefficient $>10^{5} \mathrm{~cm}^{-1} \cdot{ }^{25} \mathrm{In}$ CdTe solar cells, the back contact interface layer plays a vital role in carrier transport, and the development of high-quality back contact is essential to improve the device performance. ${ }^{26}$ Due to its deep work function, preparation of low resistance and stable ohmic contact presents a major technology challenge for 
CdTe PV devices. ${ }^{27}$ To make a good contact and reduce the contact barrier and contact resistance on CdTe, several techniques have been employed such as back surface modification or insertion of a hole transport layer (HTL) between the CdTe and a metallic contact. The CdTe back surface can be modified by doping with $\mathrm{Cu}$ or etching the CdTe surface to create a Te-rich layer. ${ }^{28-30}$ The etching procedure generally consists of exposing the polycrystalline CdTe surface to an acidic alcoholic solution of bromine or iodine that selectively reacts with $\mathrm{Cd}$ atoms and leaves Te on the surface. $^{31,32}$ Other (HTL) materials applied between CdTe and a metallic contact include iron pyrite, carbon nanotubes, copper doped ZnTe, nanocomposite $(\mathrm{CuS})_{x}(\mathrm{ZnS})_{1-x}$, and copper NWs with graphene. ${ }^{33-40}$ The formation or the deposition of Te layer is essential for the preparation of highly efficient CdTe photovoltaics. For example, the deposition of elemental Te by thermal evaporation has also improved the device performance. ${ }^{41}$ The use of length controlled Te NWs offers the important potential to develop point contacts at the back contact of p-type polycrystalline CdTe, in such a way as to allow for efficient hole extraction through an otherwise passivated interface.

Here, we report the synthesis and characterization of Te NWs with uniform length and diameter distributions using a colloidal hot injection method. During synthesis, tellurium oxide $\left(\mathrm{TeO}_{2}\right)$ is the source of Te, $\mathrm{L}$-ascorbic acid is used as a reducing agent, ethylene glycol as a solvent, and polyvinylpyrrolidone (PVP) serves as a surfactant. The synthesized Te NWs have hexagonal crystal structure with active vibrational modes at 117.0 and $136.1 \mathrm{~cm}^{-1}$ corresponding to $\mathrm{A}_{1}$ and $\mathrm{E}_{2}$ modes. Implementing these Te NWs to fabricate CdTe solar cell back contacts yield significantly improved open-circuit voltage $\left(V_{\mathrm{oc}}\right)$ and fill factor (FF) compared to standard devices. The best cell using Te NWs has an efficiency of $14.6 \%\left(V_{\text {oc }} 846 \mathrm{mV}\right.$, FF $77.8 \%$ ), an $11 \%$ improvement over the control device which converts at an efficiency of $13.1 \%$ ( $V_{\text {oc }} 825 \mathrm{mV}, \mathrm{FF} 74.0 \%$ ). These results are quite good for a film stack with CdS as the window layer, which limits the current density to about $75 \%$ of what can be obtained using a CdSe or $\mathrm{MgZnO}_{x}$ front window layer. ${ }^{42}$

\section{Experimental methods}

\section{Materials}

Tellurium oxide $\left(\mathrm{TeO}_{2} \geq 99 \%\right.$ ) was obtained from Alfa Aesar, polyvinylpyrrolidone (PVP, Molecular wt. 40000 ), L-ascorbic acid $(\geq 99 \%)$ and ethylene glycol were obtained from Fisher Scientific, and potassium hydroxide $(\mathrm{KOH} \geq 99 \%)$ was obtained from Sigma Aldrich are of analytical grade and used without further purification.

\section{Synthesis procedure}

The synthesis of Te NWs is based on a slightly modified version of the procedure reported by Yang et al. previously. ${ }^{24}$ In a typical synthesis of Te NWs, $1.05 \mathrm{mmol}(0.167 \mathrm{gm})$ of tellurium oxide $\left(\mathrm{TeO}_{2}\right), 2.98 \mathrm{mmol}(0.167 \mathrm{gm})$ of potassium hydroxide $(\mathrm{KOH})$ and $0.1 \mathrm{gm}$ of polyvinylpyrrolidone (PVP) were dissolved in $15 \mathrm{~mL}$ ethylene glycol (EG) under vigorous magnetic stirring to form a milky white solution. The temperature of the flask was set to $120{ }^{\circ} \mathrm{C}$ at which the solution becomes transparent yellow. In the meantime, $1.42 \mathrm{mmol}$ (0.2496 gm) of ascorbic acid was dissolved in $0.75 \mathrm{~mL}$ of deionized water at $80{ }^{\circ} \mathrm{C}$ to form an aqueous ascorbic acid solution. When the reaction temperature reached to $120{ }^{\circ} \mathrm{C}$, the ascorbic acid solution was rapidly injected resulting the change in color of the reactants to opaque black. The reaction was continued for $21 \mathrm{~h}$ at $120{ }^{\circ} \mathrm{C}$ in a dinitrogen $\left(\mathrm{N}_{2}\right)$ environment to complete the synthesis of high quality Te NWs. The heating mantle was removed, and the flask temperature was lowered to room temperature using a cold-water bath. The product was centrifuged and cleaned with deionized water three to five times. The obtained NW product was dried and stored as a powder in a glove box in $\mathrm{N}_{2}$ environment for characterization and application.

\section{Tellurium NWs as a back-contact interface layer}

To test Te NWs as a back contact to CdTe solar cells, we used CdS/CdTe film stacks deposited by Willard and Kelsey Solar Group, via vapor transport deposition (VTD) onto $\mathrm{TEC}^{\mathrm{TM}}-15 \mathrm{M}$ (Pilkington N.A.) glass substrates. The CdTe devices were activated by $\mathrm{CdCl}_{2}$ treatment at $390{ }^{\circ} \mathrm{C}$ for $30 \mathrm{~min}$ in dry air environment to advance grain growth, release interfacial strain and facilitate intermixing at CdS/CdTe interface. ${ }^{43}$ The standard back contact was prepared using thermally evaporated copper (3 nm) and gold $(40 \mathrm{~nm})$ followed by annealing at $150{ }^{\circ} \mathrm{C}$ for 35 min. Similarly, a solution-based copper treatment was carried out using a procedure reported previously. ${ }^{44,45}$ To prepare devices using Te NWs as the HTL, $40 \mu \mathrm{L}$ solution of Te NWs $\left(10 \mathrm{mg} \mathrm{mL}^{-1}\right)$ was dropped and spun onto the Cu-doped CdTe at 1000 and $3500 \mathrm{rpm}$ for 10 and $50 \mathrm{~s}$ respectively. Devices were then annealed at $150{ }^{\circ} \mathrm{C}$ for $1 \mathrm{~min}$, and $\mathrm{Au}(40 \mathrm{~nm})$ was thermally evaporated to complete the back contact. The completed CdTe device was scribed using a $532 \mathrm{~nm}$ laser to produce approximately 24 cells of area $0.06 \mathrm{~cm}^{2} .^{46}$

\section{Results and discussion}

\section{Synthesis and characterization of Te NWs}

Fig. 1 displays the SEM images of Te NWs prepared with varying amounts of PVP and ascorbic acid. PVP acts as a structural directing agent and stabilizing agent, ${ }^{47}$ and therefore plays a vital role in the formation of Te NWs with uniform morphology. Along with PVP, the alkaline condition aids the growth of Te NWs. Fig. 1(a) and (b) shows the SEM images of Te NWs prepared using $0.1 \mathrm{gm}$ of PVP and $0.75 \mathrm{~mL}$ of ascorbic acid solution. The synthesized Te NWs are uniform in length and diameter. Fig. 2(a) and (b) shows the diameter and length distribution of Te NWs with $0.1 \mathrm{gm}$ of PVP and $0.75 \mathrm{~mL}$ of ascorbic acid.

From SEM image Fig. 1(a) and statistical analysis Fig. 2(a) and (b), the average length and diameter of the nanowires are $1.15 \pm 0.03 \mu \mathrm{m}$ and $41.0 \pm 3.0 \mathrm{~nm}$ respectively. The Te NWs have very narrow size distribution with standard deviation $\pm 0.03 \mu \mathrm{m}$ in length and $\pm 3.0 \mathrm{~nm}$ in diameter. In this case, the aspect ratio of Te NW is $28.0 \pm 2.7$. Upon increasing the 

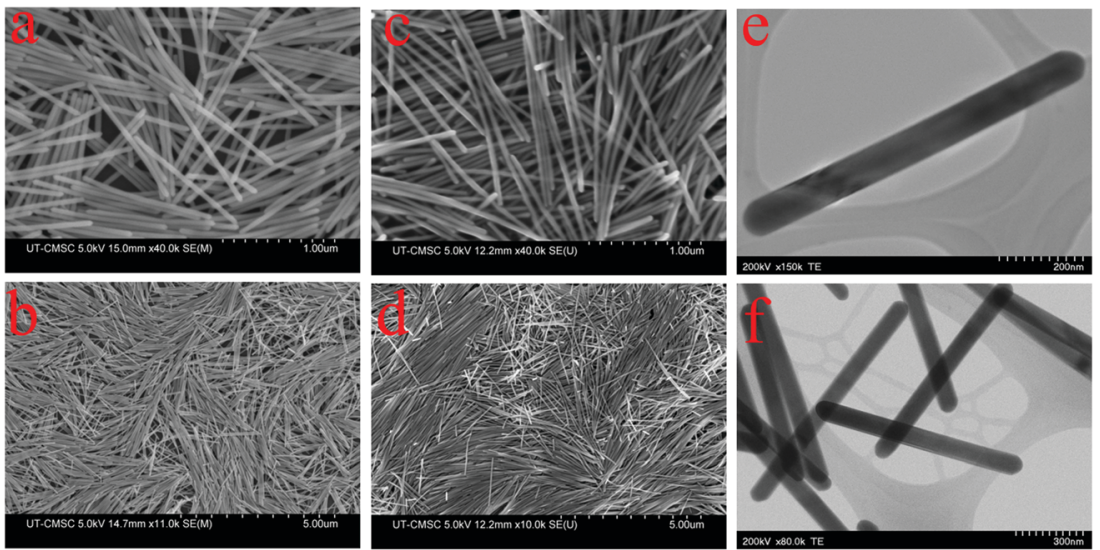

Fig. 1 SEM images of Te NWs obtained from $\mathrm{TeO}_{2}$ with ethylene glycol at $120{ }^{\circ} \mathrm{C}$ (a) and (b) $0.1 \mathrm{gm} \mathrm{PVP}$ and $0.75 \mathrm{~mL}$ ascorbic acid (c) and (d) $0.2 \mathrm{gm} \mathrm{PVP}$ and $1.5 \mathrm{~mL}$ ascorbic acid and (e) and (f) STEM images of Te NWs obtained from $0.1 \mathrm{gm}$ PVP and $0.75 \mathrm{~mL}$ ascorbic acid.
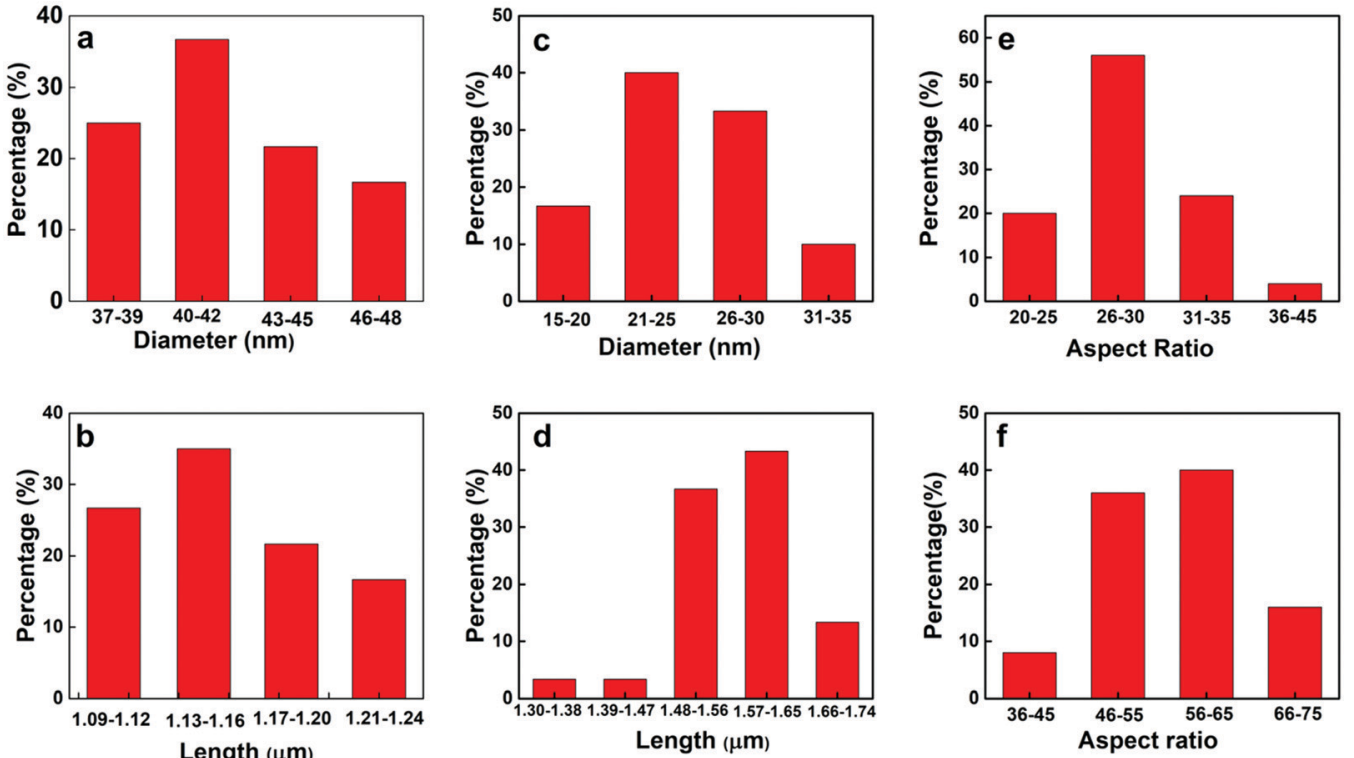

Fig. 2 Diameter, length and aspect ratio distribuiton of Te NWs at $120{ }^{\circ} \mathrm{C}$ for $21 \mathrm{~h}$. (a), (b) and (e) $0.1 \mathrm{~g} \mathrm{PVP}$ and $0.75 \mathrm{~mL}$ ascorbic acid (c), (d) and (f) $0.2 \mathrm{~g}$ PVP and $1.5 \mathrm{~mL}$ ascorbic acid.

amount of PVP to $0.2 \mathrm{~g}$ and ascorbic acid to $1.5 \mathrm{~mL}$, Te NWs of uniform length and diameter were again obtained, however with decreased NW diameter and increased NW length. The SEM images are shown in Fig. 1(c) and (d). The average length and diameter of Te NWs are $1.54 \pm 0.07 \mu \mathrm{m}$ and $27.8 \pm 3.7 \mathrm{~nm}$ respectively. The aspect ratio of Te NWs for this case is $55.4 \pm$ 1.1. Fig. 2(c) and (d) shows the diameter and length distribution of Te NWs with $0.2 \mathrm{gm}$ of PVP and $1.5 \mathrm{~mL}$ of ascorbic acid. Further, we calculated the aspect ratio of the individual NWs, and plotted the graphs for aspect ratios for two synthesis conditions as shown in Fig. 2(e) and (f). In case of the NWs synthesized by using $0.1 \mathrm{~g}$ PVP and $0.75 \mathrm{~mL}$ ascorbic acid, $\sim 56 \%$ of NWs have aspect ratio in the range of $26-30$ while for $0.2 \mathrm{~g}$ PVP and $1.5 \mathrm{~mL}$ ascorbic acid, $\sim 40 \%$ of the NWs have aspect ratio in the range of $56-65$ and $\sim 36 \%$ are in the range of 46-55. The increased in aspect ratio is due to the role of these
PVP and ascorbic acid as directing agent and reducing agent. ${ }^{11}$ Fig. 1(e) and (f) shows the scanning transmission electron microscope (STEM) images of Te NWs formed with $0.1 \mathrm{gm}$ PVP and $0.75 \mathrm{~mL}$ ascorbic acid. It reveals that NWs are highly crystalline with uniform length and diameter. The unusual anisotropic crystal structure of Te consists of 1D helical chain of covalently bound atoms parallel to the $c$-axis. ${ }^{48}$ Previously, Zhong et al. used a solvothermal technique to synthesize Te NWs with diameter $30 \pm 2 \mathrm{~nm}$ and length $10 \pm 1 \mu \mathrm{m}$ using ethylene glycol as a solvent. ${ }^{11}$ Qian et al. reported the synthesis of Te NWs with diameter 4-9 $\mathrm{nm}$ and lengths up to hundreds of $\mu \mathrm{m}$ and Wang et al. reported Te NWs with diameter $40 \mathrm{~nm}$ with lengths in several microns using hydrazine through hydrothermal method. ${ }^{14,15}$ In our case, we demonstrated the successful control of the aspect ratio of Te NWs, from 28.0 to 55.4, by varying the amount of ascorbic acid and PVP. The amount of 
surfactant and reducing agent are the key parameters to prepare the desired nanowires during synthesis. Potassium hydroxide to control alkalinity helps PVP to act fully as a surfactant for the synthesis of Te NWs. Here, we developed the Te NWs with uniform length and diameter using hot injection colloidal method with small deviation in length with standard deviations of $0.03 \mu \mathrm{m}$ and $0.07 \mu \mathrm{m}$ respectively for two cases.

Fig. 3(a) displays the typical XRD pattern of synthesized Te NWs performed in the ' $2 \theta$ ' range of $20-70^{\circ}$. The diffraction peaks obtained from MDI JADE closely match with the diffraction peaks measured for our as-synthesized Te NWs. Based on the standard powder diffraction file, synthesized Te NWs are in hexagonal phase with lattice constants $a=b=0.4454 \mathrm{~nm}$ and $c=0.5924 \mathrm{~nm}$. The high intensity obtained in the diffraction pattern signifies the purity of the material. Similarly, Fig. 3(b) shows the Raman spectrum of Te NWs using a $632.8 \mathrm{~nm} \mathrm{He}-\mathrm{Ne}$ laser source with bands at 117.0, 136.1 and $265.9 \mathrm{~cm}^{-1}$. The mode with a vibration $117.0 \mathrm{~cm}^{-1}$ corresponds to $\mathrm{A}_{1}$ mode, $136.1 \mathrm{~cm}^{-1}$ corresponds to $\mathrm{E}_{2}$ mode and the bands at
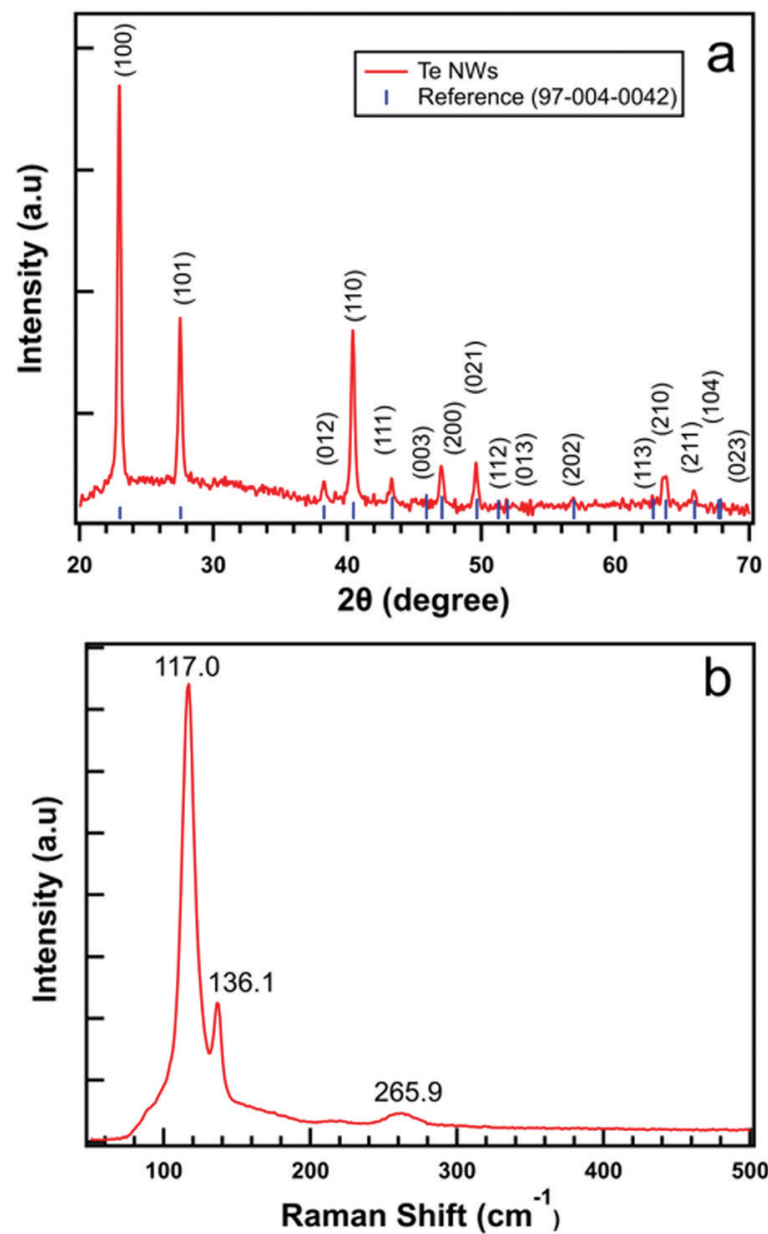

Fig. 3 (a) X-ray diffraction pattern of synthesized Te NWs obtained from $\mathrm{TeO}_{2}$, possess hexagonal crystal structure and the sharp intensity peaks of the pattern indicate pure-phase crystalline material. The reference lines (PDF \#97-004-0042) was obtained from MDI JADE software, and (b) Raman spectroscopy measurement of Te NWs using a $632.8 \mathrm{~nm}$ excitation from a $\mathrm{He}-\mathrm{Ne}$ laser with $0.1 \mathrm{gm}$ PVP and $0.75 \mathrm{~mL}$ ascorbic acid.
$265.9 \mathrm{~cm}^{-1}$ assigned to the second-order spectra of the Te NWs which agrees closely with previous reports. ${ }^{49,50}$

Next, we investigated and analyzed the effect of Te NWs as a HTL on CdTe PV devices. Tellurium manifests as a p-type semiconductor, and has been reported with a majority carrier concentration of $2.65 \times 10^{18} \mathrm{~cm}^{-3}$, Hall mobility of $8.99 \mathrm{~cm}^{2} \mathrm{~V}^{-1} \mathrm{~S}^{-1}$, and resistivity of $0.26 \Omega \mathrm{cm} .{ }^{51}$ The insertion of a Te layer as a HTL on CdTe surface is to make a $\mathrm{p} / \mathrm{p}^{+}$interface with the CdTe absorber layer. The formation of Te on the back surface reduces the potential barrier height for hole transport between the CdTe and the back metal electrode. ${ }^{32}$ Fig. 4 displays SEM images of the CdTe surface after $\mathrm{CdCl}_{2}$ treatment, (a) without and (b) with Te NWs applied. Fig. 4(b) clearly shows a CdTe surface covered with Te NWs. Fig. 4(c) shows the cross-sectional SEM image of CdS/CdTe solar cells with the Te NWs HTL and thickness of Te NWs layer is approximately $100 \mathrm{~nm}$, and Fig. 4(d) shows the schematic diagram of the device structure.

Fig. 5 shows the $J-V$ characteristics and external quantum efficiencies (EQEs) of CdS/CdTe solar cells prepared with standard back contacts and prepared using Te NWs as an interface layer in between the $\mathrm{Cu}$ and $\mathrm{Au}$. The back-contact designs tested here involved $\mathrm{Cu} / \mathrm{Au}$ (evaporated metallic $\mathrm{Cu}$ ), $\mathrm{Cu} / \mathrm{TeNWs} / \mathrm{Au}, \mathrm{CuCl}_{2} / \mathrm{Au}$ (solution $\mathrm{CuCl}_{2}$ ), and $\mathrm{CuCl}_{2} / \mathrm{TeNWs} / \mathrm{Au}$. The solid and dashed lines correspond to light and dark measurements respectively. In the case of the $\mathrm{Cu} / \mathrm{Au}$ back contact, the best device has an efficiency $(\eta)$ of $13.1 \%$ with $V_{\text {oc }} 825 \mathrm{mV}, J_{\mathrm{sc}}$ $21.6 \mathrm{~mA} \mathrm{~cm}^{-2}$, and FF of $74.0 \%$. When a Te NW film was used as the HTL $(\mathrm{Cu} / \mathrm{TeNWs} / \mathrm{Au})$, the best device efficiency was $14.0 \%$ with a $V_{\mathrm{oc}}$ of $837 \mathrm{mV}, J_{\mathrm{sc}}$ of $21.6 \mathrm{~mA} \mathrm{~cm} \mathrm{~cm}^{-2}$ and $\mathrm{FF}$ of $77.7 \%$. For $\mathrm{Cu} / \mathrm{TeNWs} / \mathrm{Au}$ back contact devices, the average device efficiency was increased by about $7 \%$ as compared to $\mathrm{Cu} / \mathrm{Au}$ back contacts with significant improvement on FF. Similarly, with solutionbased copper $\left(\mathrm{CuCl}_{2} / \mathrm{Au}\right)$, the back contact, best cell has an efficiency $(\eta)$ of $14.0 \%$ with $V_{\mathrm{oc}} 841 \mathrm{mV}, J_{\mathrm{sc}} 22.1 \mathrm{~mA} \mathrm{~cm}{ }^{-2}$, and FF of $75.4 \%$. With Te NWs $\left(\mathrm{CuCl}_{2} / \mathrm{TeNWs} / \mathrm{Au}\right)$, the best device efficiency was $14.6 \%$ with $V_{\mathrm{oc}}$ of $846 \mathrm{mV}, J_{\mathrm{sc}}$ of $22.3 \mathrm{~mA} \mathrm{~cm} \mathrm{~cm}^{-2}$

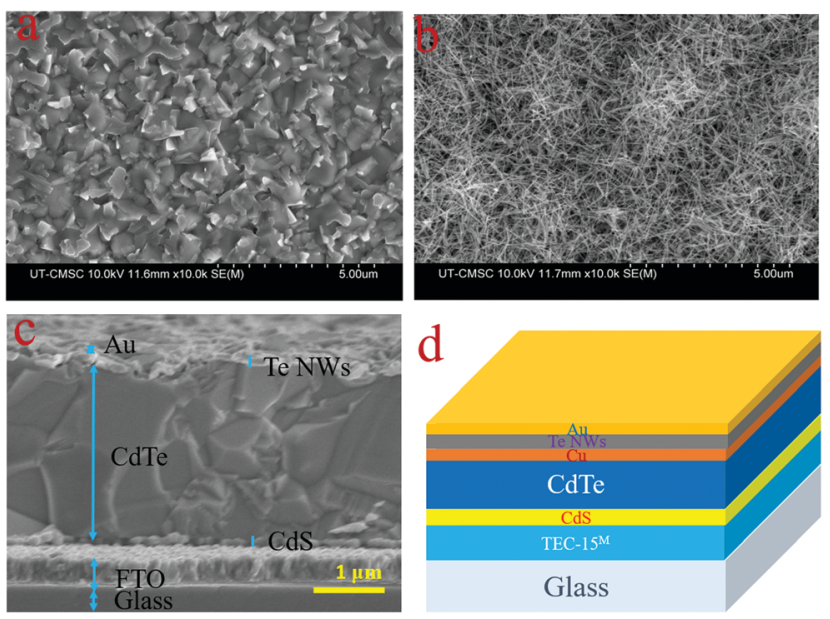

Fig. 4 SEM images of $\mathrm{CdCl}_{2}$ treated CdTe (a) top surface w/o Te NWs and (b) top surface with Te NWs (c) cross-sectional image with Te NWs as HTL and (d) schematic diagram of the device structure. 

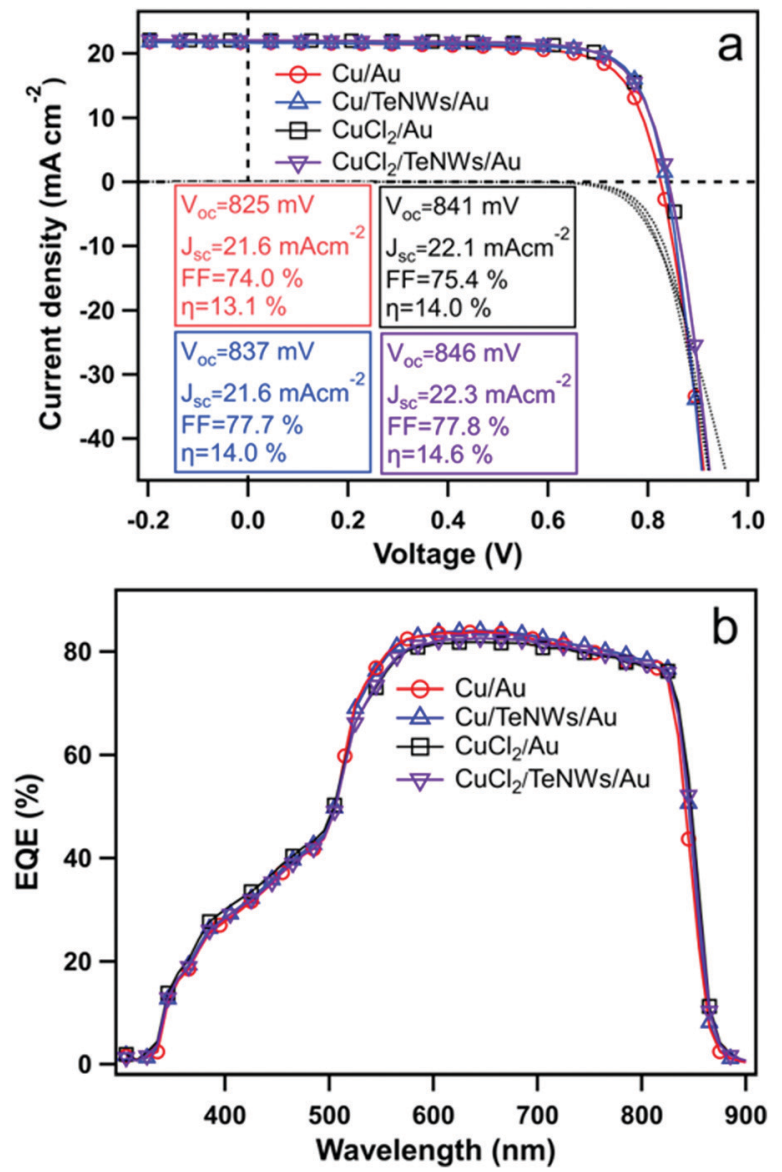

Fig. 5 (a) $J-V$ characteristics and (b) external quantum efficiency (EQE) of $\mathrm{CdTe}$ solar cells with $\mathrm{Cu} / \mathrm{Au}, \mathrm{Cu} / \mathrm{TeNW} / \mathrm{Au}, \mathrm{CuCl}_{2} / \mathrm{Au}$ and $\mathrm{CuCl}_{2} / \mathrm{TeNWs} /$ $\mathrm{Au}$ back contacts respectively. The solid and dotted lines in Fig. 5(a) represent $J-V$ curves under illumination and dark conditions respectively.

and $\mathrm{FF}$ of $77.8 \%$. We have observed that managing the $\mathrm{Cu}$ concentration and diffusion plays a critical role in performance, and we therefore believe that the use of $\mathrm{CuCl}_{2}$ solution to introduce the $\mathrm{Cu}$ enables better control than the thermal evaporation of a thin $\mathrm{Cu}$ layer. ${ }^{45}$ The average device efficiency was increased by about $4 \%$ as compared to the $\mathrm{CuCl}_{2} / \mathrm{Au}$ back contact. The average device parameters from twenty cells of each type of back contact are shown in Table 1. By introducing Te NWs as an interface layer, an improvement of open circuit voltage and fill factor was observed. The $J_{\mathrm{sc}}$ obtained from EQE measurement matches closely with $J_{\mathrm{sc}}$ obtained from $J-V$ measurement.

Tellurium as a buffer layer between CdTe and a metal contact has been shown to reduce the downward band bending and mitigate a Schottky barrier formed at the back contact. ${ }^{52}$ A Te buffer layer on CdTe can also be deposited by evaporation. Watthage et al. reported the best cell efficiency of $13.8 \%$ $\left(V_{\mathrm{oc}}=839 \mathrm{mV}\right.$, and $\left.\mathrm{FF}=75.6 \%\right)$ and average efficiency of $13.3 \%$ with $80 \mathrm{~nm}$ thermally evaporated Te followed by $3 \mathrm{~nm}$ $\mathrm{Cu}$ and $40 \mathrm{~nm} \mathrm{Au} .^{31}$ Previously, Liang et al. have also applied the $\mathrm{Cu}$ NWs with graphene as a back contact interface layer to CdTe photovoltaics, and they reported the device efficiency to be $12.1 \%$ while the control was $9.1 \%$ with $\mathrm{Cu}$ thin film. ${ }^{40}$ Similarly, Major et al. have used ZnO NWs instead of conventional $\mathrm{ZnO}$ thin film as a front contact material for CdTe solar cells. They reported photoconversion efficiency of 9.53\% when using $\mathrm{ZnO}$ NWs, whereas use of a planar ZnO buffer layer had a device efficiency of $9.99 \% .^{53}$ Compared to these results, we observed $>11 \%$ increase in device efficiency by using Te NWs compared to $\mathrm{Cu} / \mathrm{Au}$ back contacts. Thus, a thin layer of Te NWs seems to be more effective to enhance the device efficiency which we attribute to the lowering of contact resistance and barrier height.

The $J-V$ behavior of solar cells can be described by using single exponential diode equation as,

$$
J=J_{0} \exp \left[\frac{q}{A k_{\mathrm{B}} T}\left(V-J R_{\mathrm{s}}\right)+G_{\mathrm{sh}} V-J_{\mathrm{L}}\right]
$$

where $J_{0}$ is the reverse saturation current, $q$ is an electronic charge, $A$ is an ideality factor, $k_{\mathrm{B}}$ is Boltzmann's constant, $T$ is absolute temperature, $R_{\mathrm{S}}$ is series resistance, $G_{\mathrm{sh}}$ is shunt conductance and $J_{\mathrm{L}}$ is the load current density which is equal to short circuit current density $\left(J_{\mathrm{sc}}\right)$. To analyze the solar cell parameters using dark current, we take $J_{\mathrm{L}}=J_{\mathrm{sc}}=0$. By utilizing the experimental dark $J-V$ curves and above diode equation, we calculated and compared the solar cell parameters like ideality factor, series resistance, and dark saturation current for CdS/ $\mathrm{CdTe}$ device with back contacts $\mathrm{Cu} / \mathrm{Au}, \mathrm{Cu} / \mathrm{TeNWs} / \mathrm{Au}, \mathrm{CuCl}_{2} / \mathrm{Au}$ and $\mathrm{CuCl}_{2} / \mathrm{TeNWs} / \mathrm{Au}$ respectively.

Fig. 6(a) displays dark $J-V$ curves on a linear scale for various back contacts. In case of a $\mathrm{Cu} / \mathrm{Au}$ back contact, we can see higher back barrier which explains the low fill factor of these cells compared to other back contacts. As evidenced from the dark $J-V$ curves with Te NWs, we can see the improvement in the $J-V$ behavior qualitatively. Fig. 6(b) shows the plot of $\mathrm{d} J / \mathrm{d} V$ versus bias voltage $(V)$, and it indicates negligible shunt conductance (close to zero) in the devices under dark conditions. Thus, the term $G_{\text {sh }}$ and $J_{\mathrm{L}}$ were taken to be zero for these dark $J-V$ curves. Similarly, Fig. 6(c) shows the plot of $\mathrm{d} V / \mathrm{d} J v s . J^{-1}$ from which we estimated the values of $R_{\mathrm{s}}$ and $A$. The values of the $R_{\mathrm{s}}$ and $A$ were obtained from the $y$-intercept and slope of the linear fit as shown in the Fig. 6(c). Similarly, using these values

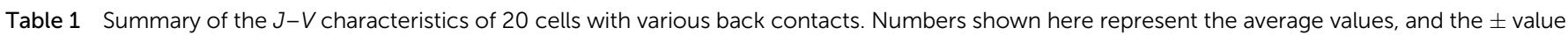
represents the standard deviation (SD) of the measured device parameters

\begin{tabular}{|c|c|c|c|c|c|c|}
\hline Back-contacts & $V_{\mathrm{oc}}(\mathrm{mV})$ & $J_{\mathrm{sc}}\left(\mathrm{mA} \mathrm{cm}{ }^{-2}\right)$ & FF (\%) & $\eta(\%)$ & $R_{\mathrm{s}}\left(\mathrm{ohms} \mathrm{cm}^{2}\right)$ & $R_{\text {shunt }}\left(\right.$ ohms $\left.\mathrm{cm}^{2}\right)$ \\
\hline $\mathrm{Cu} / \mathrm{Au}$ & $818 \pm 5$ & $21.6 \pm 0.3$ & $72.8 \pm 0.8$ & $12.9 \pm 0.2$ & $3.5 \pm 1.2$ & $1737 \pm 228$ \\
\hline $\mathrm{Cu} / \mathrm{TeNWs} / \mathrm{Au}$ & $835 \pm 1$ & $21.5 \pm 0.1$ & $77.3 \pm 0.2$ & $13.9 \pm 0.1$ & $3.8 \pm 0.1$ & $3504 \pm 228$ \\
\hline $\mathrm{CuCl}_{2} / \mathrm{Au}$ & $838 \pm 2$ & $22.0 \pm 0.1$ & $74.8 \pm 0.9$ & $13.8 \pm 0.2$ & $4.3 \pm 0.1$ & $2983 \pm 466$ \\
\hline $\mathrm{CuCl}_{2} / \mathrm{TeNWs} / \mathrm{Au}$ & $844 \pm 1$ & $22.0 \pm 0.2$ & $77.2 \pm 0.2$ & $14.3 \pm 0.1$ & $4.0 \pm 0.3$ & $3781 \pm 225$ \\
\hline
\end{tabular}



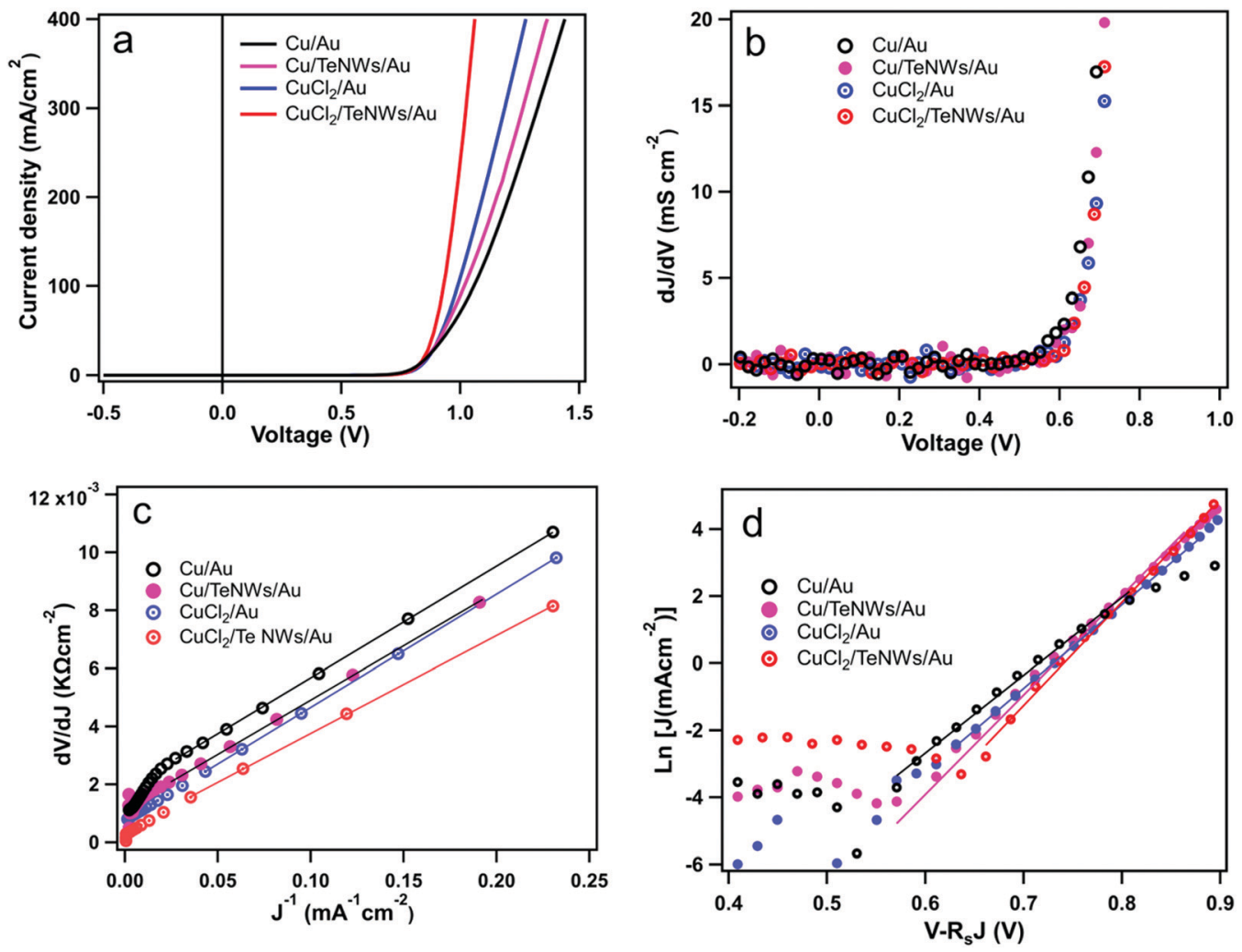

Fig. 6 Analysis of dark J-V curves with back contacts $\mathrm{Cu} / \mathrm{Au}, \mathrm{Cu} / \mathrm{TeNWs} / \mathrm{Au}, \mathrm{CuCl}_{2} / \mathrm{Au}$ and $\mathrm{CuCl}_{2} / \mathrm{TeNWs} / \mathrm{Au}$ (a) dark J-V curve (b) shunt characterization (c) plot of $\mathrm{d} V / \mathrm{d} J$ versus $\mathrm{l} / \mathrm{J}$ for calculation of $A$ and $R_{\mathrm{s}}(\mathrm{d})$ plot of $\ln (\mathrm{J})$ versus $V-R_{\mathrm{s}} \mathrm{J}$ for calculation of $n$ and $J_{0}$. The solid lines represent the corresponding fitted lines in (c) and (d).

Table 2 Device parameters (ideality factor, series resistance, and dark saturation current) obtained by analyzing dark J-V curves (Fig. 2) based on Fig. 6

\begin{tabular}{lllll}
\hline Device parameters & $\mathrm{Cu} / \mathrm{Au}$ & $\mathrm{Cu} / \mathrm{TeNWs} / \mathrm{Au}$ & $\mathrm{CuCl}_{2} / \mathrm{Au}$ & 1.56 \\
\hline$A\left(\mathrm{~d} V / \mathrm{d} J v s . J^{-1}\right)$ & 1.54 & 1.48 & 0.76 & 1.36 \\
$R_{\mathrm{s}}\left(\mathrm{d} V / \mathrm{d} J v s . J^{-1}\right)\left(\Omega \mathrm{cm}^{2}\right)$ & 1.83 & 1.21 & 1.44 & 0.48 \\
$A\left(\ln J v s . V-R_{\mathrm{S}} J\right)$ & 1.60 & 1.35 & $1.2 \times 10^{-9}$ & 1.3 \\
$\left.J_{0}\left(\ln J v s . V-R_{\mathrm{S}} J\right)(\mathrm{mA} \mathrm{cm})^{-2}\right)$ & $3.5 \times 10^{-8}$ & $3.6 \times 10^{-10}$ & & $1.2 \times 10^{-10}$
\end{tabular}

of $R_{\mathrm{s}}$, we plotted $\ln [J]$ versus $\left[V-R_{S} J\right]$ as shown in Fig. $6(\mathrm{~d})$ to estimate reverse saturation current density and ideality factor. The values of $R_{\mathrm{s}}, A$ and $J_{0}$ for all back contacts obtained from Fig. 6 are presented in Table 2 .

The value of $A$ with $\mathrm{Cu} / \mathrm{Au}$ back contact is about 1.54 while with Te NWs, its value is lowered to 1.48. Also, the reverse saturation current density $\left(J_{0}\right)$ is $3.5 \times 10^{-8} \mathrm{~mA} \mathrm{~cm}^{-2}$ for $\mathrm{Cu} / \mathrm{Au}$ and with Te NWs its value is $3.6 \times 10^{-10} \mathrm{~mA} \mathrm{~cm}^{-2}$. The values of ideality factor and reverse saturation current are in close agreement with a previous report for $\mathrm{CdS} / \mathrm{CdTe}$ device with standard back contact $(\mathrm{Cu} / \mathrm{Au}) .{ }^{54}$ Similarly, the ideality factor and $J_{0}$ are improved for $\mathrm{CuCl}_{2}$ treated devices with Te NWs. Thus, the interfacial layer helped to lower the value of the reverse saturation current density indicating better diode behavior of these CdS/ CdTe solar cells. In a device, $A$ and $J_{0}$ depend one each other while the value of $J_{0}$ influences the open-circuit voltage of the device. In case of $\mathrm{CuCl}_{2} / \mathrm{TeNWs} / \mathrm{Au}$, it has the lowest value of the $J_{0}$ and highest value of $V_{\mathrm{oc}}$, and lower value of the $J_{0}$ indicates the reduced recombination at the back interface.
Here, Te NWs are used as a back-buffer layer to CdS/CdTe devices which reduces the contact barrier and improves the transport of holes towards the back electrode. In CdS/CdTe devices, $J_{\mathrm{sc}}$ is low due to the parasitic absorption of blue photons by CdS layer compared to CdSe/CdTe or MZO/CdTe devices. However, the device efficiency that we have obtained here are comparable to previously published reports for $\mathrm{CdS} / \mathrm{CdTe}$ devices. ${ }^{55}$ In ref. 55 , the authors have used very thin $(50 \mathrm{~nm}) \mathrm{CdS}$ layer than in our case $(120 \mathrm{~nm})$ yielding higher $J_{\mathrm{sc}}\left(25.1 \mathrm{~mA} \mathrm{~cm} \mathrm{~cm}^{-2}\right)$ while they have lower FF (74.5\%) compared to our results (Table 1). Here, our ultimate goal is to utilize orientation- and contactcontrolled Te NWs as current conduits to channel holes through an insulated and passivated CdTe back contact interface and enhance the FF and $V_{\text {oc }}$ of the device.

\section{Conclusions}

We have succesfully synthesized 1-D Te NWs of uniform lengths and diameters via hot injection collodial method. The lengths 
and diameters of the Te NWs can be controlled using the amount of ascorbic acid and PVP. A typical length and diameter of Te NWs with $0.1 \mathrm{gm}$ PVP and $0.75 \mathrm{~mL}$ ascorbic acid were $1.15 \pm 0.10 \mu \mathrm{m}$ and $41.0 \pm 3.0 \mathrm{~nm}$ respectively. The synthesized Te NWs have hexagonal crystal sturcutre with active modes of vibrations at $117.0 \mathrm{~cm}^{-1}$ and $136.1 \mathrm{~cm}^{-1}$ for $\mathrm{A}_{1}$ and $\mathrm{E}_{2}$ modes. Te NWs thin films were utilized as a hole transport layer to CdS/ CdTe solar cells to enhance the open-circuit voltage, fill factor, and power conversion efficiency. On using these Te NWs as an interface layer, average device efficiency of more than 20 cells was increased by $\sim 11.0 \%$ (absolute $1.4 \%$ ) compared to standard $\mathrm{Cu} / \mathrm{Au}$ back contact.

\section{Disclaimer}

The views and conclusions contained herein are those of the authors and should not be interpreted as necessarily representing the official policies or endorsements, either expressed or implied, of Air Force Research Laboratory or the U.S. Government.

\section{Conflicts of interest}

There are no conflicts to declare.

\section{Acknowledgements}

This material is based on research sponsored by Air Force Research Laboratory under agreement number FA9453-18-20037. The U.S. Government is authorized to reproduce and distribute reprints for Governmental purposes notwithstanding any copyright notation thereon. We also thank Willard and Kelsey Solar Group for providing CdS/CdTe device stacks.

\section{References}

1 M. S. Gudiksen, et al., Growth of nanowire superlattice structures for nanoscale photonics and electronics, Nature, 2002, 415(6872), 617.

2 S. E. Steinvall, et al., Multiple morphologies and functionality of nanowires made from earth-abundant zinc phosphide, Nanoscale Horiz., 2020, 5(2), 274-282.

3 Y. Li, et al., Solution-processed one-dimensional $\mathrm{CsCu}_{2} \mathrm{I}_{3}$ nanowires for polarization-sensitive and flexible ultraviolet photodetectors, Mater. Horiz., 2020, 7(6), 1613-1622.

$4 \mathrm{X}$. Duan, et al., Indium phosphide nanowires as building blocks for nanoscale electronic and optoelectronic devices, Nature, 2001, 409(6816), 66-69.

5 Y. Huang, X. Duan and C. M. Lieber, Nanowires for integrated multicolor nanophotonics, Small, 2005, 1(1), 142-147.

6 A. V. Naumov, Erratum: To the article "Selenium and Tellurium: state of the markets, the crisis, and its consequences" by A. V. Naumov, vol. 54, no. 3-4, pp. 197-200, July, 2010, Metallurgist, 2011, 54(9), 714.

7 S. Lin, et al., Tellurium as a high-performance elemental thermoelectric, Nat. Commun., 2016, 7(1), 10287.
8 J.-W. Liu, et al., Rapid Microwave-Assisted Synthesis of Uniform Ultralong Te Nanowires, Optical Property, and Chemical Stability, Langmuir, 2010, 26(13), 1372-11377.

9 Y. Zhang, et al., Low-cost writing method for self-powered paper-based UV photodetectors utilizing $\mathrm{Te} / \mathrm{TiO}_{2}$ and $\mathrm{Te} / \mathrm{ZnO}$ heterojunctions, Nanoscale Horiz., 2019, 4(2), 452-456.

10 Y. Wang, et al., Two-dimensional ferroelectricity and switchable spin-textures in ultra-thin elemental Te multilayers, Mater. Horiz., 2018, 5(3), 521-528.

11 B. N. Zhong, et al., Controlled solvothermal synthesis of single-crystal tellurium nanowires, nanotubes and trifold structures and their photoelectrical properties, CrystEngComm, 2017, 19(20), 2813-2820.

$12 \mathrm{X}$. Wu, et al., Controlled synthesis of multi-morphology Te crystals by a convenient Lewis acid/base-assisted solvothermal method, J. Nanopart. Res., 2012, 14(8), 1009.

$13 \mathrm{Z}$. He and S.-H. Yu, Large scale synthesis of tellurium nanoribbons in tetraethylene pentamine aqueous solution and the stability of tellurium nanoribbons in ethanol and water, J. Phys. Chem. B, 2005, 109(48), 22740-22745.

14 H.-S. Qian, et al., High-Quality Luminescent Tellurium Nanowires of Several Nanometers in Diameter and High Aspect Ratio Synthesized by a Poly(Vinyl Pyrrolidone)-Assisted Hydrothermal Process, Langmuir, 2006, 22(8), 3830-3835.

$15 \mathrm{Z}$. Wang, et al., Formation of single-crystal tellurium nanowires and nanotubes via hydrothermal recrystallization and their gas sensing properties at room temperature, J. Mater. Chem., 2010, 20(12), 2457-2463.

16 U. K. Gautam and C. N. R. Rao, Controlled synthesis of crystalline tellurium nanorods, nanowires, nanobelts and related structures by a self-seeding solution process, J. Mater. Chem., 2004, 14(16), 2530-2535.

17 G. Xi, et al., Synthesis, Characterization, and Growth Mechanism of Tellurium Nanotubes, Cryst. Growth Des., 2005, 5(1), 325-328.

18 Q. Wang, et al., Fabrication and growth mechanism of selenium and tellurium nanobelts through a vacuum vapor deposition route, J. Phys. Chem. C, 2007, 111(35), 12926-12932.

19 P. Mohanty, J. Park and B. Kim, Large scale synthesis of highly pure single crystalline tellurium nanowires by thermal evaporation method, J. Nanosci. Nanotechnol., 2006, 6(11), 3380-3383.

20 Z.-H. Lin, Z. Yang and H.-T. Chang, Preparation of fluorescent tellurium nanowires at room temperature, Cryst. Growth Des., 2007, 8(1), 351-357.

21 R. R. Silva, et al., Facile synthesis of tellurium nanowires and study of their third-order nonlinear optical properties, J. Braz. Chem. Soc., 2017, 28(1), 58-67.

22 N. R. Jana, L. Gearheart and C. J. Murphy, Wet Chemical Synthesis of High Aspect Ratio Cylindrical Gold Nanorods, J. Phys. Chem. B, 2001, 105(19), 4065-4067.

23 C. K. Senthil kumaran, et al., Synthesis and Characterization of Selenium Nanowires, ISRN Nanotechnol., 2011, 2011, 4.

$24 \mathrm{H}$. Yang, et al., Environmentally Benign Synthesis of Ultrathin Metal Telluride Nanowires, J. Am. Chem. Soc., 2014, 136(29), 10242-10245. 
25 D. Bonnet and P. Meyers, Cadmium-telluride-Material for thin film solar cells, J. Mater. Res., 1998, 13(10), 2740-2753.

26 G. K. Liyanage, et al., The role of back buffer layers and absorber properties for $>25 \%$ efficient CdTe solar cells, ACS Appl. Energy Mater., 2019, 2(8), 5419-5426.

27 A. L. Fahrenbruch, Ohmic contacts and doping of CdTe, Sol. Cells, 1987, 21(1-4), 399-412.

28 D. Bätzner, et al., Development of efficient and stable back contacts on CdTe/CdS solar cells, Thin Solid Films, 2001, 387, 51-154.

29 S. Demtsu, D. Albin and J. Sites, Role of copper in the performance of CdS/CdTe solar cells, 2006, 1, 523-526.

30 D. W. Niles, et al., A photoemission determination of the band diagram of the Te/CdTe interface, J. Appl. Phys., 1995, 77(9), 4489-4493.

31 S. C. Watthage, et al., Selective Cd Removal From CdTe for High-Efficiency Te Back-Contact Formation, IEEE J. Photovolt., 2018, 8(4), 1125-1131.

32 E. Bastola, et al., Wet chemical etching of cadmium telluride photovoltaics for enhanced open-circuit voltage, fill factor, and power conversion efficiency, J. Mater. Res., 2019, 34(24), 3988-3997.

33 T. Gessert, et al., Development of Cu-doped ZnTe as a backcontact interface layer for thin-film CdS/CdTe solar cells, J. Vac. Sci. Technol., A, 1996, 14(3), 806-812.

34 N. Romeo, et al., A highly efficient and stable CdTe/CdS thin film solar cell, Sol. Energy Mater. Sol. Cells, 1999, 58(2), 209-218.

35 A. B. Phillips, et al., Wiring-up carbon single wall nanotubes to polycrystalline inorganic semiconductor thin films: lowbarrier, copper-free back contact to CdTe solar cells, Nano Lett., 2013, 13(11), 5224-5232.

36 W. Xia, et al., Te/Cu bi-layer: A low-resistance back contact buffer for thin film CdS/CdTe solar cells, Sol. Energy Mater. Sol. Cells, 2014, 128, 411-420.

37 E. Bastola, K. P. Bhandari and R. J. Ellingson, Application of composition controlled nickel-alloyed iron sulfide pyrite nanocrystal thin films as the hole transport layer in cadmium telluride solar cells, J. Mater. Chem. C, 2017, 5(20), 4996-5004.

38 K. P. Bhandari, et al., Iron pyrite nanocrystal film serves as a copper-free back contact for polycrystalline CdTe thin film solar cells, Sol. Energy Mater. Sol. Cells, 2015, 140, 108-114.

39 K. K. Subedi, et al., Nanocomposite $(\mathrm{CuS})_{x}(\mathrm{ZnS})_{1-x}$ thin film back contact for CdTe solar cells: Toward a bifacial device, Sol. Energy Mater. Sol. Cells, 2018, 186, 227-235.

$40 \mathrm{~J}$. Liang, et al., Novel $\mathrm{Cu}$ nanowires/graphene as the back contact for CdTe solar cells, Adv. Funct. Mater., 2012, 22(6), 1267-1271.
41 D. W. Niles, et al., Evaporated Te on CdTe: A vacuumcompatible approach to making back contact to CdTe solar cell devices, Prog. Photovoltaics Res. Appl., 1996, 4(3), 225-229.

42 A. H. Munshi, et al., Polycrystalline CdTe photovoltaics with efficiency over $18 \%$ through improved absorber passivation and current collection, Sol. Energy Mater. Sol. Cells, 2018, 176, 9-18.

43 K. V. Krishna and V. Dutta, Effect of in situ $\mathrm{CdCl}_{2}$ treatment on spray deposited CdTe/CdS heterostructure, J. Appl. Phys., 2004, 96(7), 3962-3971.

44 D. Mao, et al., Correlative impurity distribution analysis in cadmium telluride (CdTe) thin-film solar cells by ToF-SIMS 2D imaging, Sol. Energy Mater. Sol. Cells, 2016, 157, 65-73.

45 E. Bastola, et al., Doping of CdTe using CuCl2 Solution for Highly Efficient Photovoltaic Devices, 2019 IEEE 46th Photovoltaic Specialists Conference (PVSC), 2019, pp. 1846-1850.

46 A. B. Phillips, et al., High speed, intermediate resolution, large area laser beam induced current imaging and laser scribing system for photovoltaic devices and modules, Rev. Sci. Instrum., 2016, 87(9), 093708.

47 Z. Zhang, B. Zhao and L. Hu, PVP protective mechanism of ultrafine silver powder synthesized by chemical reduction processes, J. Solid State Chem., 1996, 121(1), 105-110.

48 G. Kresse, J. Furthmüller and J. Hafner, Theory of the crystal structures of selenium and tellurium: the effect of generalizedgradient corrections to the local-density approximation, Phys. Rev. B: Condens. Matter Mater. Phys., 1994, 50(18), 13181.

49 H. Park, et al., Aqueous chemical synthesis of tellurium nanowires using a polymeric template for thermoelectric materials, CrystEngComm, 2015, 17(5), 1092-1097.

$50 \mathrm{G}$. Li, et al., Solvothermal synthesis of polycrystalline tellurium nanoplates and their conversion into single crystalline nanorods, RSC Adv., 2014, 4(2), 954-958.

51 Y. Li, et al., Effects of evaporated tellurium back buffer layer on cdte solar cell, Chalcogenide Lett., 2019, 16(11), 535-544.

52 T. Song, A. Moore and J. R. Sites, Te Layer to Reduce the CdTe Back-Contact Barrier, IEEE J. Photovolt., 2018, 8(1), 293-298.

53 J. D. Major, et al., Development of $\mathrm{ZnO}$ nanowire based CdTe thin film solar cells, Sol. Energy Mater. Sol. Cells, 2017, 160, 107-115.

54 K. P. Bhandari, et al., Thin film iron pyrite deposited by hybrid sputtering/co-evaporation as a hole transport layer for sputtered CdS/CdTe solar cells, Sol. Energy Mater. Sol. Cells, 2017, 163, 277-284.

55 J. Britt and C. Ferekides, Thin-film CdS/CdTe solar cell with 15.8\% efficiency, Appl. Phys. Lett., 1993, 62(22), 2851-2852. 